\title{
Action comprehension in aphasia: linguistic and non-linguistic deficits and their lesion correlates
}

\author{
Ayşe Pınar Saygın ${ }^{\mathrm{a}, *}$, Stephen M. Wilson ${ }^{\mathrm{b}}$, Nina F. Dronkers ${ }^{\mathrm{c}, \mathrm{d}}$, Elizabeth Bates ${ }^{\mathrm{a},}{ }^{\dagger}$ \\ ${ }^{a}$ Department of Cognitive Science, University of California, San Diego, 9500 Gilman Drive 0515, La Jolla, CA 92093-0515, USA \\ ${ }^{\mathrm{b}}$ Neuroscience Interdepartmental Program, University of California, Los Angeles, CA, USA \\ ${ }^{\mathrm{c}}$ VA Northern California Health Care System, Martinez, USA \\ ${ }^{\mathrm{d}}$ Departments of Neurology and Linguistics, University of California, Davis, CA, USA
}

Received 28 August 2003; received in revised form 25 April 2004; accepted 30 April 2004

\begin{abstract}
We tested aphasic patients' comprehension of actions to examine processing deficits in the linguistic and non-linguistic domains and their lesion correlates. Twenty-nine left-hemisphere injured patients and 18 age-matched control subjects matched pictured actions (with the objects missing) or their linguistic equivalents (printed sentences with the object missing) to one of two visually-presented pictures of objects. Aphasic patients performed poorly not only in the linguistic domain but also in the non-linguistic domain. A subset of the patients, largely consisting of severe and non-fluent aphasics, showed a greater deficit in the linguistic domain compared with the non-linguistic domain and across the patient group, deficits in the linguistic and non-linguistic domains were not tightly correlated. Poor performance in pantomime interpretation was associated with lesions in the inferior frontal, premotor and motor cortex, a portion of somatosensory cortex, and the caudate, while poor reading comprehension of actions was associated with lesions around the anterior superior temporal lobe, the anterior insula and the anterior portion of the inferior parietal lobe. Lesion size did not correlate with deficits. The lesion results for pantomime interpretation deficits demonstrate that lesions in the frontal component of the human analog of the "mirror neuron system" are associated with deficits in non-linguistic action understanding. For reading comprehension deficits, the lesion correlates are brain areas known to be involved in linguistic tasks including sentence processing and speech articulation; the parietal lesion site may also correspond to a subpart of the human mirror neuron system. These results indicate that brain areas important for the production of language and action are also recruited in their comprehension. Similar findings have been reported in electrophysiological and neuroimaging studies. Our findings now also lend neuropsychological support to an embodied view of brain organization for action processing.
\end{abstract}

(C) 2004 Elsevier Ltd. All rights reserved.

Keywords: Pantomime; Reading; Mirror neurons; Action understanding; Lesion-symptom mapping; Embodiment

\section{Introduction}

While aphasia is primarily characterized by disturbance of language functions following brain injury, patients have been observed to also exhibit impairments in nonverbal domains, revealed by tasks such as associating pictures with corresponding objects (De Renzi, Pieczuro, \& Vignolo, 1968), colors with pictures (De Renzi, Faglioni, Scotti, \& Spinnler, 1972), and environmental sounds with pictures (e.g., Saygin, Dick, Wilson, Dronkers, \& Bates, 2003a; Spinnler \& Vignolo, 1966). In particular, aphasic patients' deficits in using and recognizing signs, gestures and pantomime have

\footnotetext{
* Corresponding author. Tel.: +1 858534 1148; fax: +1 8585346788 . E-mail address: asaygin@cogsci.ucsd.edu (A.P. Saygın).

$\dagger$ Elizabeth Bates, Professor of Cognitive Science at the University of California, San Diego, passed away December 13, 2003.
}

been examined in numerous studies (e.g., Bell, 1994; Duffy \& Duffy, 1981; Gainotti \& Lemmo, 1976; Goodglass \& Kaplan, 1963; Pickett, 1974; Varney, 1978; Wang \& Goodglass, 1992).

In the present study, we examined aphasic patients' comprehension of visually presented action stimuli in both linguistic and non-linguistic domains. We used a variant of a classical neuropsychological paradigm commonly used to test comprehension in aphasic patients: an object selection task. We had a two-alternative forced-choice (2AFC) design and asked patients to choose the object that best matched visually presented stimuli containing action information. In both the linguistic and the non-linguistic domains, the associated objects upon which the actions should be carried out were removed from the stimuli; thus subjects matched either a sentence missing its object (such as "he is licking the ..."), or a picture missing its object (such as a picture of a 
boy licking an invisible ice-cream cone) to the corresponding object (in this case, the ice-cream cone). We used static black and white drawings of pantomimed actions in our design (like Seron, van der Kaa, Remitz, \& van der Linden, 1979) because they are more appropriate visual matches to written text stimuli which are also static in nature.

We had two main goals in this study: (1) to test linguistic and non-linguistic action comprehension at the same time, using the same task, on the same patients, and with stimuli as closely matched as possible, (2) to conduct lesion-symptom mapping analyses using voxel-based lesion symptom mapping (VLSM; Bates et al., 2003b) to identify the lesion correlates of action comprehension deficits.

Regarding the first goal, relationships between linguistic and non-linguistic deficits in aphasic patients are important to examine because they shed light on whether aphasia is a domain-specific disorder which affects only language, or is part of a larger deficit which affects other domains as well. Such questions have been asked since the early days of neurology. Finkelnburg (1870) was the first to propose what is known as the "asymbolia" hypothesis: he suggested that a single underlying factor was common to both the language impairments in aphasia and the deficits in nonverbal domains that these patients exhibit. This idea received some support from subsequent pioneers in neurology as well (e.g., Goldstein, 1948; Head, 1926). On the other hand, it does not seem plausible that aphasia is completely reducible to a strong version of asymbolia, especially given that dissociations in performance between linguistic and non-linguistic domains can be encountered in individual patients.

Even though nonverbal deficits in aphasia have been of interest to researchers for a long time, it has been difficult to assess whether the linguistic and the non-linguistic deficits patients exhibit are related to each other. First, performance on language processing and on non-linguistic tasks must be explored in the same patients. Furthermore, task and stimulus-level factors should be as closely matched as possible. Considerations such as these motivate the first goal of the present study: to contrast linguistic and non-linguistic comprehension of action information in aphasia by comparing performance in the two domains in the same patients more directly than in previous studies. Previous studies seeking correlations between patients' performance in various language tests and various action comprehension tests do exist, although stimuli and tasks have often not been closely matched across the two domains. While some of these studies found correlations between language impairments and non-linguistic action processing impairments in aphasic patients (e.g., Duffy \& Duffy, 1981; Pickett, 1974; Seron et al., 1979; Varney, 1978, 1982), others found largely uncorrelated performance (e.g., Bell, 1994; Goodglass \& Kaplan, 1963; Kimura, 1977).

With regards to the second goal, although it has been known since the early days of neurology that left-hemisphere lesions can often cause receptive and/or expressive disorders in both language and action domains (i.e., aphasic and apraxic disorders) and that patients with right hemisphere injury will rarely exhibit such impairments, the precise lesion sites leading to aphasic and apraxic deficits remain quite unclear. Specifically, results on lesion correlates of impairments in action, pantomime and gesture comprehension deficits are few, and not entirely consistent. Heilman and colleagues have reported that apraxic patients with posterior lesions have more trouble in comprehending the meaning of pantomimes (Heilman, Rothi, \& Valenstein, 1982; Rothi, Heilman, \& Watson, 1985) and have suggested that posterior parietal regions of the cortex may mediate the production and comprehension of purposeful movements (see also De Renzi, Faglioni, Scarpa, \& Crisi, 1986; Kertesz, Ferro \& Shewan, 1984). On the other hand, Ferro, Martins, Mariano, and Castro Caldas (1983) reported that while gesture recognition impairments were most commonly associated with parietal lesions in chronic stages of brain damage, in acute stages it was the patients with left frontal and basal ganglia damage who showed deficiencies, but unfortunately this study had a rather small sample size. Other studies failed to find reliable lesion sites associated with deficits (e.g., Schnider, Hanlon, Alexander, \& Benson, 1997; Wang \& Goodglass, 1992). Recently, Tranel, Kemmerer, Adolphs, Damasio, and Damasio (2003) used more novel lesion-mapping methods and reported that lesions in the left premotor/prefrontal and parietal cortex and in the white matter underlying the left posterior middle temporal cortex are implicated in deficits in tasks which were designed to tap into conceptual knowledge for actions.

There is also a substantial literature on the related question of brain areas differentially involved in the naming of actions versus objects, and/or the processing of verbs versus nouns. Many researchers have argued that left frontal areas are differentially involved in the processing of actions or verbs. For example, in a PET study using a lexical decision task, Perani, Cappa, Schnur, and Tettamanti (1999) found that verbs activated left dorsolateral frontal cortex more than nouns. However, other studies have failed to find significant differences; for instance, Tyler, Russell, Fadili, and Moss (2001), using carefully matched stimuli, did not find any regions differentially involved in the lexical decision or semantic processing of nouns versus verbs. Hillis, Tuffiash, Wityk, and Barker (2002) reported that damage or hypoperfusion in precentral and middle temporal gyri were associated with action naming deficits in patients with acute left hemisphere injury, while for object naming, middle temporal and superior temporal gyri were associated with impairment. However, for comprehension of action and object words, they did not find separate sites; impairments were associated with superior temporal lesions. Hillis et al. suggested in light of this finding that only the naming of actions, rather than semantic knowledge, may be localized to left frontal cortex.

Given the diverse results which have been reported in the literature, we wanted to use VLSM, a quantitative lesionsymptom mapping technique, to contribute to identifying 
lesion correlates of action comprehension in aphasia in linguistic and non-linguistic domains.

In addition to these two major goals, we had some other points in mind in our design: In line with earlier studies (e.g., Varney, 1978), we also addressed the effect of semantic competition in both domains in order to see if processing in the two domains is similarly modulated by higher-level conceptual constraints. In addition, following Seron et al. (1979), Wang and Goodglass (1992) and Bell (1994), we also used distracters that were related to the targets in the way they may be handled, to see if there are differential effects of this kind of competition (previous researchers termed these morphological, perceptual or motoric distracters; here we refer to these as "affordance-based" distracters; see Gibson, 1977).

\section{Methods}

\subsection{Participants}

Patients were voluntary participants recruited from the community in San Diego, CA or the VA Northern California Health Care System (VANCHCS) in Martinez, CA, and were paid US\$ 25.00 for their participation. Twenty-nine left-hemisphere injured patients with varying types and severity of aphasia participated in the experiment. All aphasic patients were administered the Western Aphasia Battery (WAB; Kertesz, 1979) and were diagnosed as Anomic ( $N$ $=9)$, Broca's $(N=12)$, or Wernicke's aphasics $(N=8)$. In this sample, we did not have patients with other types of aphasia (e.g., global, conduction). More detail is provided in Table 1. Computerized tomography (CT) or magnetic resonance imaging (MRI) scans and medical records of all patients were evaluated by a neurologist at the time of enrolment into our program, and only patients with unilateral lesions due to a single cerebrovascular accident were included. Exclusionary criteria included non-native English proficiency, as well as a diagnosis or suspicion of hearing difficulties, dementia, head trauma, tumors, multiple infarcts or other neurological conditions. We carefully monitored for patients with any diagnosed or suspected visual problems, including agnosia. No patients were excluded on this basis. Subjects with corrected-to-normal vision were allowed to participate. For this particular study, patients were also administered a subtest of the Minnesota Test for Differential Diagnosis of Aphasia (MTDDA; Schuell, 1965) in order to assess their ability to match single words to pictures, so as to exclude patients with severe single word reading deficits. It was planned that patients who scored below $75 \%$ in this $2 \mathrm{AFC}$ task would not be allowed to participate, but

Table 1

Characteristics of aphasic patients

\begin{tabular}{|c|c|c|c|c|}
\hline Initials & Age & Aphasia type & AQ & Lesion site \\
\hline B.E. & 25 & Broca's & 71.6 & Frontal, temporal, parietal, insula, basal ganglia \\
\hline B.K. & 56 & Anomic & 84.4 & Basal ganglia, insula \\
\hline C.H. & 67 & Anomic & 92.2 & Basal ganglia \\
\hline D.C. & 64 & Broca's & 74.8 & Frontal, insula, basal ganglia \\
\hline D.D. & 57 & Broca's & 18.9 & Temporal, parietal, frontal, insula \\
\hline D.F. & 47 & Broca's & 49.6 & Temporal, parietal, frontal, insula \\
\hline F.Y. & 78 & Wernicke's & 64.1 & Inferior parietal, small region on superior temporal \\
\hline H.K. & 63 & Wernicke's & 47.6 & Frontal, medial temporal, insula, subcortical \\
\hline H.K. & 75 & Broca’s & $\mathrm{n} / \mathrm{a}$ & Frontal, temporal, parietal, head of caudate \\
\hline H.M. & 73 & Broca's & 26.7 & Frontal, temporal, parietal \\
\hline J.B. & 67 & Broca's & 13.8 & MCA-territory, acute scan shows expanding frontal lesion \\
\hline J.C. & 82 & Anomic & 91.1 & N/A-acute scan, shows no lesion boundaries \\
\hline J.H. & 63 & Anomic & 92.4 & Frontal, tip of anterior temporal \\
\hline J.Q. & 77 & Broca's & 11.2 & Frontal, temporal, parietal, insula \\
\hline J.S. & 52 & Broca's & 48.8 & Frontal, temporal, parietal \\
\hline J.T. & 78 & Wernicke's & 31.7 & Temporal \\
\hline J.W. & 73 & Anomic & 90.9 & Temporal, parietal \\
\hline K.W. & 65 & Anomic & 98.0 & Frontal \\
\hline L.R. & 57 & Anomic & 79.2 & Frontal, temporal, parietal \\
\hline M.B. & 51 & Broca's & 31.0 & Frontal, insular and subcortical extension, parietal \\
\hline P.B. & 76 & Anomic & 98.0 & Medial frontal \\
\hline P.P. & 51 & Wernicke's & 78.0 & Frontal, temporal, parietal, insula \\
\hline R.S. & 75 & Wernicke's & 48.7 & Temporal, inferior parietal \\
\hline S.A. & 77 & Anomic & 66.7 & Frontal, anterior temporal \\
\hline S.S. & 78 & Broca's & 22.6 & Frontal, anterior temporal \\
\hline V.H. & 72 & Wernicke's & 78.6 & Frontal, anterior temporal \\
\hline W.G. & 83 & Wernicke's & 51.5 & Temporal, parietal \\
\hline W.R. & 59 & Broca's & 72.8 & Frontal, anterior temporal \\
\hline W.T. & 67 & Wernicke's & 73.6 & Frontal, posterior temporal \\
\hline
\end{tabular}

Patient group determined using the Western Aphasia Battery (WAB), AQ: aphasia quotient, a measure of aphasia severity, based on the WAB. Lesion summaries are based on CT or MRI scans or medical records. 
we did not encounter any such patients; indeed over $90 \%$ of our patients scored over $90 \%$ on this task, with many performing perfectly.

Age-matched controls were 18 adults aged 50-80 years, with no history of neurological, or psychiatric disorders; all had normal or corrected-to-normal vision and hearing. All were paid US\$ 25.00 for their participation. There were an additional 20 participants from UCSD, aged 18-35, who took part in two preliminary norming studies in exchange for course credit. All had normal or corrected-to-normal vision and hearing.

The study was approved by the University of California, San Diego (UCSD) and VANCHCS Human Research Protection Programs, and was performed in accordance with the ethical standards laid down in the 1964 Declaration of Helsinki. Participants gave informed consent prior to participation.

\subsection{Experimental design and materials}

A 2-within- $\times 1$-between-subjects design was used, with Domain (linguistic versus non-linguistic) and Distracter Type (semantic, affordance-based, unrelated distracters) as within-subject factors, and Subject Group (Control, Aphasic) as the between-subjects factor. In further analyses, we also included aphasia severity (AQ) and aphasia type (Anomic, Broca's, Wernicke's) as between-subjects factors.

Stimuli were black-and-white line drawings of pantomimed actions and objects, and written text. The drawings of pantomimed actions depicted people carrying out transitive actions, but with the objects removed. These stimuli were created by the first author (APS) in collaboration with an artist. Eighteen such pictures, along with three practice items, were selected from an initial set of 30 in a preliminary norming study. In the norming study, 12 subjects were shown each of the 30 pictures. They were instructed to explain the action in each picture by providing a sentence that describes the picture, and they were required to provide a verb and a noun. They were allowed to write as many as three sentences for each picture. These responses were used to select the most identifiable actions, to determine the target objects, and to determine the linguistic labels to be used in the main experiment. A list of items is provided in Table 2 along with corresponding distracters in each of the conditions.

Linguistic stimuli were (incomplete) sentences based on the most common labels provided by the subjects in the norming study. Since the target objects were missing from the picture stimuli, the objects were missing from the sentence stimuli as well. Thus sentences were of the form ' $\mathrm{He}$ is licking the ...' or 'She is sweeping with the ...'. Grammatical complexity was kept constant by putting together commonly reported nouns and verbs in 'He/she is verb-ing [preposition] the ...' constructions.

The object stimuli were digitized drawings culled from extensively normed picture databases. Naming norms for these pictures have been reported elsewhere (Szekely et al., 2004). Four line drawings of objects were matched to each action: a target, a semantically related distracter, an affordance-based distracter and an unrelated distracter. For example, depicted in Fig. 1 are the stimuli for the action licking ice-cream cone: The non-linguistic stimulus was a drawing of a person holding and licking an invisible ice-cream cone and the linguistic stimulus was the sentence fragment "he is licking the ...". The target item in each case was ice-cream cone. The semantically related distracter was the picture of a cake, an object one would eat, but normally not "lick" as depicted in the sentence (so that it was not a better fit than the target), and not hold and lick in the manner depicted in the action picture. The affordance-based distracter was a bouquet

Table 2

List of items used, along with target and distracter objects

\begin{tabular}{|c|c|c|c|c|}
\hline Action & Target & Semantic distracter & Affordance-based distracter & Unrelated distracter \\
\hline Blowing out a candle & Candle & Lamp & Cigarette & Football \\
\hline Brushing hair & Hair brush & Bow (for hair) & Knife & Boat \\
\hline Brushing teeth & Toothbrush & Dentures & Paintbrush & Pig \\
\hline Digging with a shovel & Shovel & Wheelbarrow & Guitar & Light bulb \\
\hline Drinking water from a glass & Glass & Faucet & Telescope & Cat \\
\hline Eating a burger & Burger & Salt & Football & Helicopter \\
\hline Eating an ice-cream cone & Ice-cream cone & Cake & Bouquet of flowers & Rooster \\
\hline Fencing & Foil & Mask & Umbrella & Penguin \\
\hline Playing the guitar & Guitar & Flamenco dancer & Rifle & Horse \\
\hline Playing the piano & Piano & Ballet shoes & Desk & Fish \\
\hline Raking & Rake & Leaf & Flag & Book \\
\hline Shooting with a bow and arrow & Bow and arrow & Target & Violin & Bus \\
\hline Singing into a microphone & Microphone & Television & Wrench & Onion \\
\hline Sweeping with a broom & Broom & Bucket & Double bass & Spaghetti \\
\hline Swinging a baseball bat & Baseball bat & Baseball & Frying pan & Sheep \\
\hline Talking on the telephone & Telephone & Alarm clock & Drill & Barrel \\
\hline Throwing a baseball & Baseball & Net & Light bulb & Tree \\
\hline Typing & Typewriter & Envelope & Knitting & Skateboard \\
\hline
\end{tabular}



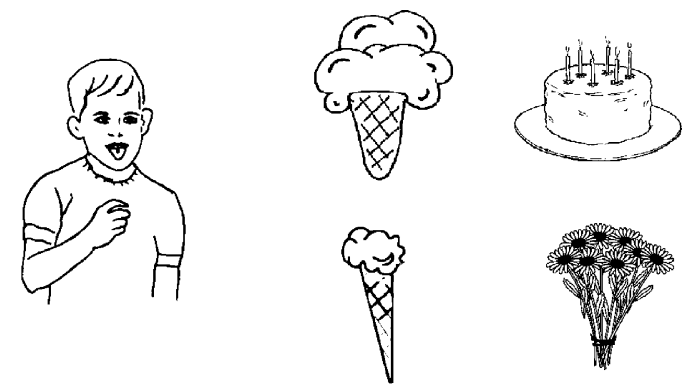

he is licking the ...
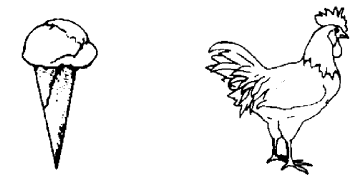

Fig. 1. Summary of the experimental design. Here, the Domain and Distracter conditions are illustrated using the action stimulus licking ice-cream cone. The left panel shows the non-linguistic (pantomime) and linguistic (text) stimuli. The linguistic stimuli were based on the most common label provided for the picture stimuli in our preliminary norming study. On the right panel, the three pairs of pictures show the target (ice cream-cone), along with the semantic (cake), affordance-based (bouquet of flowers), and unrelated (rooster) distracters for this item. In the experiment, only one of the three pairs was presented during each trial, and the two object pictures were displayed below the pantomime or text stimulus (see Section 2).

of flowers, an object one would typically hold in a manner similar to an ice-cream cone, but normally not manipulate with the mouth in the manner depicted in the picture (so that it was not a better fit than the target), and would not "lick" as depicted in the sentence. The unrelated distracter was a rooster, an object one would manipulate neither in the manner depicted in the picture, nor in the sentence.

In order to ensure that semantic relatedness was assigned appropriately across the conditions, we made use of the semantic relatedness measure latent semantic analysis (LSA; Landauer, Foltz, \& Laham, 1998). Higher LSA indices indicate higher relatedness. The average LSA index for the semantically related pairs was 0.40 , for affordance-based pairs it was 0.09 , and for unrelated pairs it was 0.006 .

To verify the affordance-based distracter assignments, we carried out another norming study in the form of a questionnaire. Eight participants were provided with pictures of target objects (objects matching the action stimuli we selected) and three other objects (the semantic, affordance-based, and unrelated distracters we assigned based on LSA). They were given detailed written instructions to rank order the three latter pictures as to how well they fulfilled the following statement in relation to the target object: this object may be held, manipulated, acted upon or interacted with in a way that is similar to the way one could hold, manipulate, act upon or interact with the target object. They were encouraged to reply based on physical properties of the objects rather than on conceptual relationships. The results of the study confirmed our choices of affordance-based distracters: these were ranked first (mean rank $=1.06$; median rank $=1)$. Semantically-related distracters were ranked second (mean rank $=2.08$; median rank $=2$ ), and unrelated distracters third (mean rank $=2.83$; median rank $=3$ ).

Over the course of the experiment, trials appeared in pseudorandomized order. Each stimulus appeared in the linguistic and non-linguistic conditions as well as with three distracter types (Fig. 1). Three separate pictures of the target object were used for each action stimulus to avoid repetition of the exact same target pictures.

\subsection{Experimental procedure}

The experiment was run on Apple iBook computers using the PsyScope experimental driver (Cohen, MacWhinney, Flatt, \& Provost, 1993). Participants sat in front of the monitor and a standard PsyScope button box was used to collect responses. The experimenter read a set of instructions to the participant, and asked him or her to complete a practice session of six trials.

The experimental design was analogous to a previous study (Saygin et al., 2003a). There were 108 experimenteradvanced trials. In each trial, subjects were presented with a two-picture display on the screen. These pictures were presented on the lower half of the screen, one on each side. After $1000 \mathrm{~ms}$, the pantomime or text stimulus was presented centrally on the upper half of the screen, above the two object pictures. This delay allowed participants enough time to process the object stimuli prior to being presented with the action stimuli. Participants pushed the button under the picture they believed matched the pantomime or sentence. Reaction time (RT) and accuracy were recorded for each trial. Participants were continuously monitored for attention to the task, and were asked at intervals whether they needed a break. The nature of each error was noted, as were any comments made during or after the experiment. Care was taken to note whether or not the participant was immediately aware of the error (as indicated by an overt verbal or physical response). Motivational feedback (e.g., "you are doing great so far", "going good") was given as often as considered necessary to keep participants engaged in the task (for aphasic patients, approximately once every 20 trials); however, this feedback did not relate any information about accuracy in a particular trial.

\subsection{Behavioral analysis}

Performance across groups was compared using repeated measures analysis of variance (ANOVA) and analysis of covariance (ANCOVA). Regression and correlation analyses were performed in order to examine the relationships between performance in the two domains. We also conducted outlier analyses (Bates, Appelbaum, Salcedo, Saygin, \& Pizzamiglio, 2003a) and cluster analyses (Sokal \& Sneath, 1973). Results were Geisser-Greenhouse corrected, where appropriate. 


\subsection{Lesion analysis}

Lesion analysis was carried out using voxel-based lesionsymptom mapping (VLSM) techniques recently developed by our group, described in Bates et al. (2003b). VLSM involves carrying out statistical analyses of the relationship between tissue damage and behavior on a voxel-by-voxel basis, and plotting the resultant statistics as color maps which depict the degree of behavioral involvement for each voxel. VLSM analyzes the relationship between behavioral data and lesion location and extent without requiring any cutoffs or grouping to be stipulated based on behavior or lesion site.

There are also limitations inherent in this kind of lesion analysis which should be noted. Firstly, the lesions of the patients in our sample do not cover the entire brain. Because all patients' lesions were restricted to the left hemisphere, we are unable to examine any hemispheric effects on action comprehension, of the sort discussed, for example, by Goldenberg (1999) in the domain of gesture perception, production and imitation. Moreover, most of the lesions in our sample resulted from infarcts of the middle cerebral artery (MCA), and hence only in MCA territory do we have sufficient sample sizes to make inferences.

Secondly, lesions almost invariably affect multiple brain areas. In lesion-symptom mapping, damage to an area may correlate with behavior because the area genuinely supports the cognitive function in question, or because the area is frequently lesioned along with some other area which is actually crucial for the function. Bates et al. (2003b) discussed the use of analyses of covariance to examine multiple areas which may potentially underlie behavioral deficits, but in the present study our sample size is not sufficient to perform such analyses. However, the lesion maps obtained by Bates et al. (2003b) for two WAB subscales do confirm that VLSM yields results which broadly conform to established locations for major linguistic functions, supporting the validity of the method.

As noted above, head CT or MRI images were obtained for each patient. For 21 of our patients, computerized lesion reconstructions to be used in lesion overlay analyses were available; the remaining lesion information reported in Table 1 was obtained from CT or MRI scans or neuroradiological reports. Lesion reconstructions were based on CT or MRI scans at least 3 weeks post-onset and were handdrawn onto 11 axial slice templates based on the atlas of DeArmond, Fusco, and Dewey (1976). The reconstructions were then entered into a Macintosh computer via electronic bitpad using in-house software. The reconstructions were performed by a board-certified neurologist with experience in neuroradiology who was blind to the behavioral deficits of the patients. Voxels were $0.5 \mathrm{~mm} \times 0.5 \mathrm{~mm}$ in-plane, with approximately $6 \mathrm{~mm}$ between slices.

At each voxel, patients were divided into two groups according to whether they did or did not have a lesion affecting that voxel. Behavioral scores were then compared for these two groups, yielding a statistic for each voxel, which was then plotted. The statistic computed in the present study was $d$, a standard measure of effect size determined by dividing the difference in group means by the pooled sample standard deviation. The $d$-maps were smoothed in-plane with a circular filter with a radius of $3.5 \mathrm{~mm}$. Voxels where fewer than five patients had lesions were excluded, as $d$ is a measure of effect size, not an inferential statistic, so values are not reliable if either of the two groups being compared is not well represented. Software to perform VLSM operates on lesion files in the popular ANALYZE image format, and is freely available online at http://crl.ucsd.edu/vlsm.

\section{Results}

Here, we report differences in accuracy and reaction time between patient and control groups, the correlation in performance across verbal and nonverbal domains, and the relationship between lesion site and processing deficits.

\subsection{Behavioral results}

We examined accuracy and reaction time (RT) for the aphasic subjects and their age-matched controls. RTs were analyzed only for correct responses and were measured from the onset of the action stimulus.

As depicted in Fig. 2a, groups differed in their overall accuracy $[F(1,45)=13.47, P=0.0006]$; aphasic patients were significantly less accurate than control subjects. There was no main effect of Domain, but a tendency for accuracy to be higher in the nonverbal (pictured) action comprehension trials $[F(1,45)=3.31, P=0.076]$. The interaction of Domain by Group $[F(1,45)=6.26, P=0.016]$ reached significance, revealing that patients made comparatively more linguistic errors than controls, as would be expected based on the fact that all patients were clinically diagnosed with aphasia.

Distracter Type had an effect on accuracy $[F(2,90)=9.66$, $P=0.0006]$ reflecting that overall, subjects made more errors when the distracters were related to the target object, compared with when they were unrelated. The effect of Distracter Type was modulated by Group $[F(2,90)=9.75, P$ $=0.0006]$, showing that patients were disproportionately affected by the distracter manipulations. The data are shown in Fig. 2b. This interaction was driven by the following effects (all Bonferroni corrected): Patients were significantly less accurate in trials with semantic distracters compared with unrelated distracters $(P<0.0001)$ and also compared with affordance-based distracters $(P<0.05)$; the effect of affordance-based distractors did not reach significance compared with unrelated distracters after correction $(P=0.16)$. For controls, the only significant effect was when affordancebased distracters were compared to unrelated distracters $(P$ $=0.012$ ). These distracter effects showed no differentiation between the linguistic and non-linguistic conditions: The interaction of Distracter Type and Domain as well as the threeway interaction of Group, Domain, and Distracter Type were 
(a)

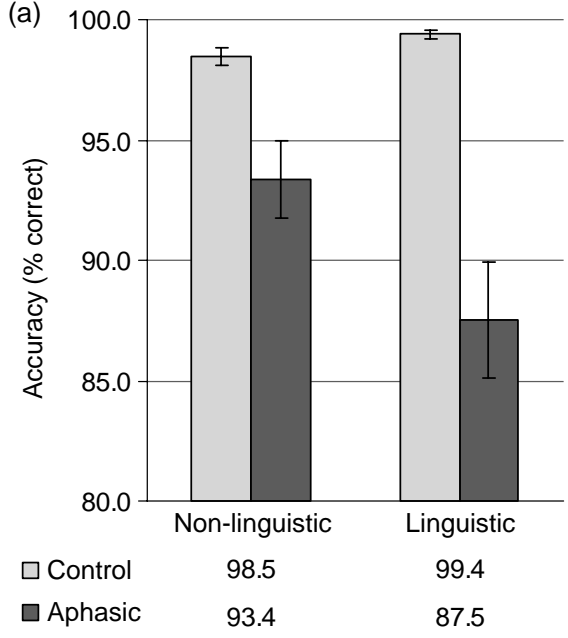

(b)

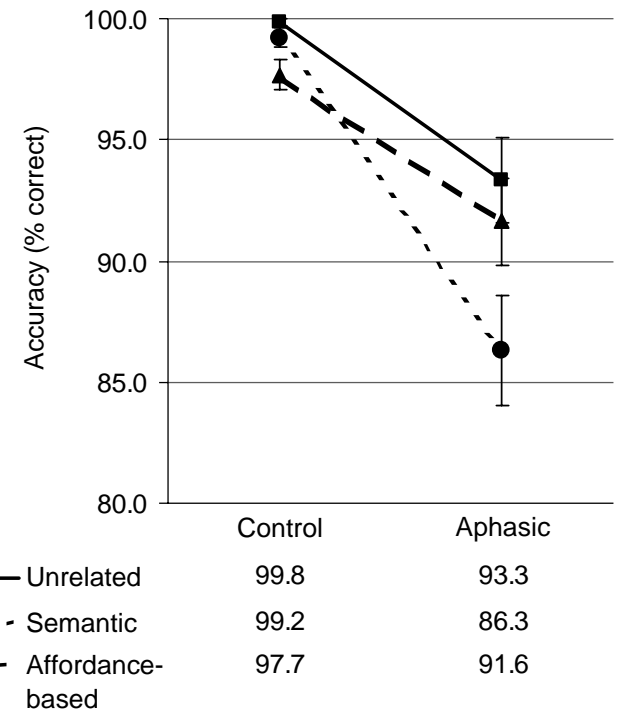

Fig. 2. Accuracy data shown across the linguistic and non-linguistic domains for the two subject groups (a). Error bars represent the standard error of the mean, in this and subsequent figures. Aphasic patients were significantly less accurate than control subjects in both linguistic and non-linguistic domains. The group by domain interaction was also significant. Accuracy data is also depicted across related and unrelated distracter conditions for the two groups (b). There was a main effect of Distracter Type and also an interaction of Distracter Type by Group, indicating that aphasic patients were disproportionately affected by the semantically related distracters.

not significant $[F(2,90)=1.12, P=0.32 ; F(2,90)=0.26$, $P=0.74]$.

We found significant differences in RT by subject group, as plotted in Fig. $3[F(1,45)=23.40, P<0.0001]$; patients responded slower than controls. There was a main effect of Domain on reaction time $[F(1,45)=13.72, P=0.0006]$ where subjects were slower to respond on the linguistic trials. There was an interaction of Domain and Group $[F(1$, $45)=11.83, P=0.0013$ ]; as can be seen in Fig. 3a, patients responded especially slowly in the linguistic domain.
There was a significant main effect of Distracter Type $[F(2,90)=8.90, P=0.0003]$, shown in Fig. 3b. Overall the slowest response was to semantic distracters and this was significant compared with unrelated distracters $(P<$ 0.0001 , all comparisons corrected) as well as affordancebased distracters $(P=0.017)$. Affordance-based distracters led to slower reaction times compared to unrelated distracters, but this reached significance only in the control group $(P=0.0018)$. Overall, Distracter Type did not interact with Group $[F(2,90)=1.15, P=0.22]$. The interaction of
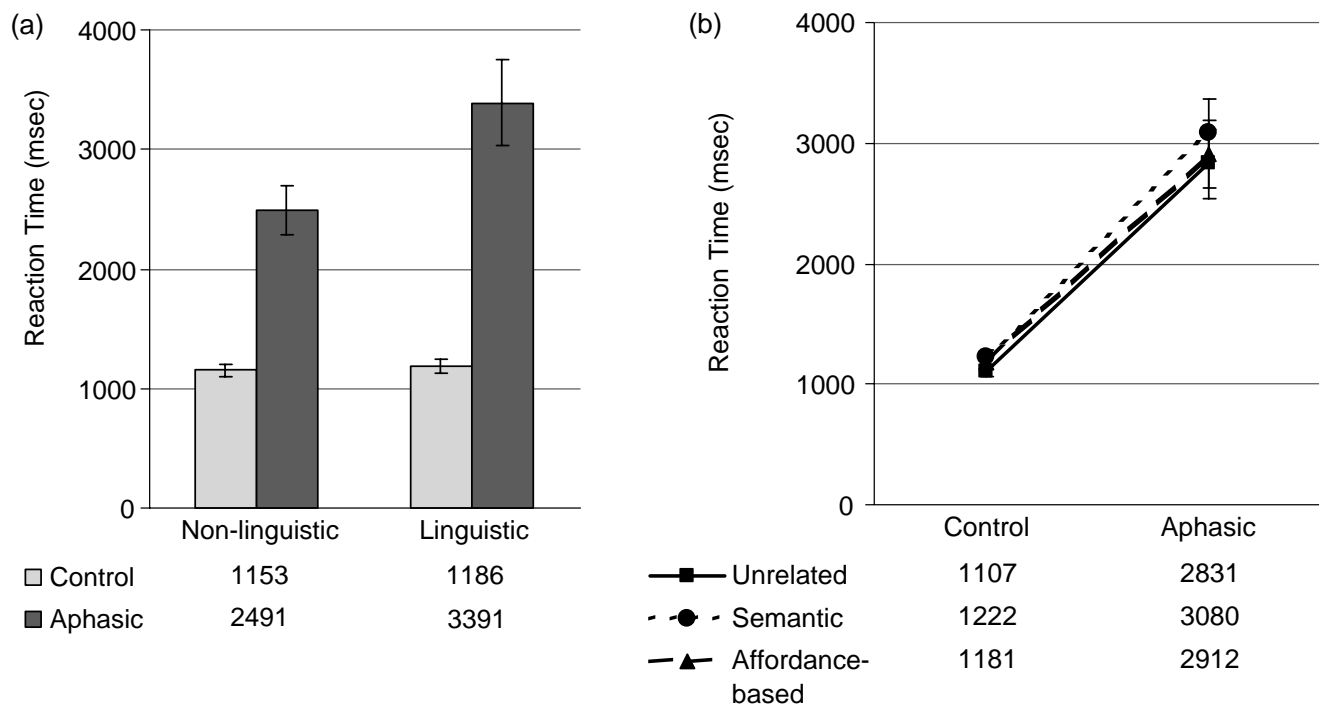

Fig. 3. Reaction time (RT) for correct responses depicted across linguistic and non-linguistic domains for the two subject groups (a). Patients with aphasia were significantly slower than controls in both domains but the RT discrepancy was larger in the linguistic domain. RT data is also shown for related and unrelated distracter conditions for the two groups (b). There was a main effect of Distracter Type; semantic distracters had the largest effect. 
Distracter and Domain as well as the three-way interaction of Group, Domain, and Distracter Type were not significant for RT $\left[F^{\prime} \mathrm{s}<1\right]$.

We next conducted analyses based only on the patients' data to see how the experimental scores were related to aphasia severity, by including aphasia quotient (AQ, a measure of aphasia severity, derived from the patients' WAB scores) as a continuous variable in our analysis. Low quotients are associated with severe aphasia and higher quotients are associated with relatively mild aphasia. Mean AQ in this sample was 61.5 (S.D. $=25.2$, range: $11.2-98.0)$. Table 1 reports AQ for each of our patients.

There were main effects of AQ $[F(1,26)=15.92, P$ $=0.0005]$ and Domain $[F(1,26)=18.24, P=0.0002]$, and an interaction of Domain by $\mathrm{AQ}[F(2,52)=10.76, P$ $=0.003]$. There was a main effect of Distracter Type $[F(2$, 52) $=14.35, P<0.0001]$ and an interaction of Distracter by AQ $[F(2,52)=5.67, P=0.011]$. The interaction of Distracter and Domain and the three-way interaction were not significant $\left[F^{\prime} \mathrm{s}<1\right]$. The significant interactions of Domain by AQ and Distracter Type by AQ reveal that the patient group's performance is related to aphasia severity; more severely affected patients were responsible for both the Domain and the Distracter Type effects. Several effects were also mirrored in the RT data: There was a main effect of AQ $[F(1,26)=12.37, P=0.0016]$, a main effect of Domain $[F(1,26)=24.53, P<0.0001]$, and an interaction with Domain and AQ $[F(1,26)=10.06, P=0.0039]$. To summarize, the severity of aphasia was seen to be a significant correlate of the relatively severe impairment the patient group exhibited in the linguistic domain and also to the relatively difficult time they had with semantic distracters.

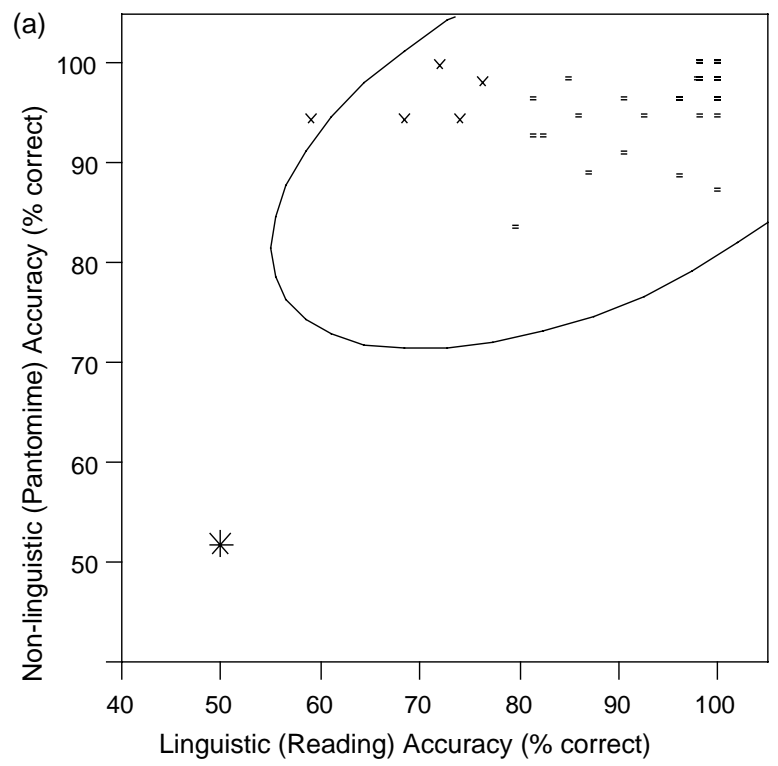

We next added the grouping variable aphasia type (Anomic, Broca's, Wernicke's, based on the WAB) to the model. In these analyses, aphasia type had no significant main effects or interactions for either accuracy or RT, but the effects reported above remained significant.

\subsection{Associations between task performance across domains, cluster and outlier analyses}

So far we saw that aphasic patients have significant deficits in both linguistic and non-linguistic action comprehension. However, this does not necessarily imply that the deficits have a common substrate.

We examined if the deficits were correlated in the two domains and at a first glance it appeared that accuracy in the linguistic and non-linguistic domains were significantly correlated in our action recognition test $[r=0.53 ; P=0.03]$. However, a closer inspection showed that this correlation was pulled by patient JB (marked with ' $*$ ' in Fig. 4a), whose performance was at chance for both domains. This patient was reliably identified as an outlier by our analyses and was singled out in cluster analyses (see below). When the correlation analysis was repeated without JB in the dataset, we found that the correlation between accuracy in linguistic and non-linguistic domains was no longer significant $[r=0.12$; $P=0.53]$.

On the other hand, a high correlation between the two domains was found for the RT data $[r=0.91 ; P<0.0001$, see Fig. 4b]. This correlation, unlike the one for accuracy, was robust and still held when the outliers were excluded from analyses $[r=0.90 ; P<0.0001]$. In the absence of a correlation in accuracy scores, we can only interpret the RT

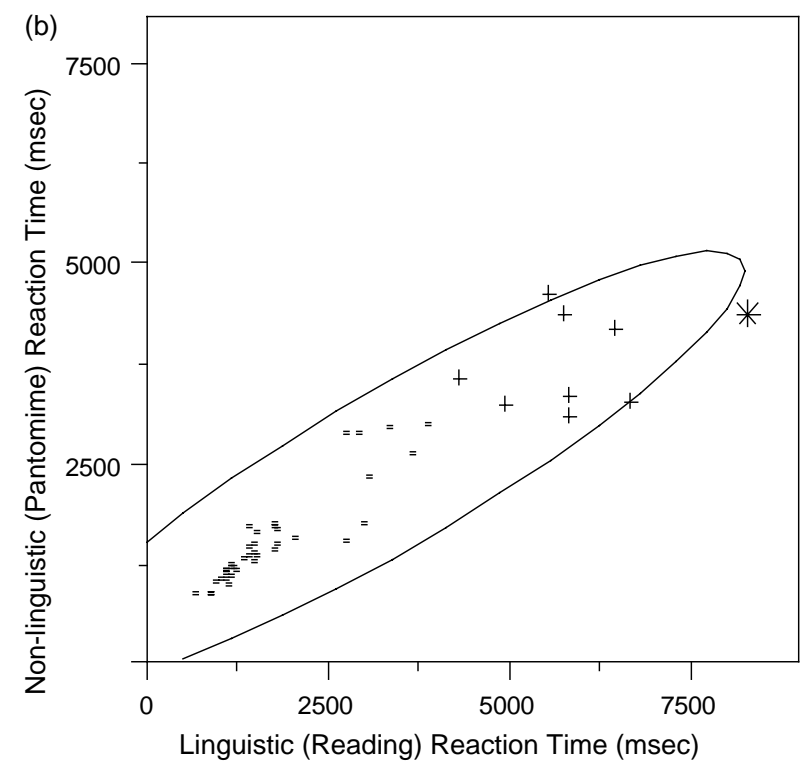

Fig. 4. Correlation of performance in the verbal and nonverbal domains within the aphasic group for (a) accuracy and (b) reaction time. Density ellipses using a confidence interval of $95 \%$ are shown. Data points outside the ellipses are outliers based in Mahalanobis distances. Cluster analysis results are also depicted with the different markers. In Fig. 5a, '*' denotes patient JB, ' $x$ ' and ' ' denote the second and third clusters. In Fig. 5b '*' denotes patient RS, '+' and '.' denote the second and third clusters. 
correlations as being due to common factors in the task such as motor planning and execution, orienting and attention, rather than being reflective of an association between the linguistic and non-linguistic domains.

In order to further explore the relative impairments in the two domains, we performed cluster analyses on the data. We first performed hierarchical clustering with cluster size 3 with the accuracy in the two domains (linguistic, nonlinguistic) as variables, a process that transforms data points into a sequence of nested partitions (Sokal \& Sneath, 1973). The clusters are plotted using different markers in Fig. 4a. Cluster 1 consists of patient JB and represents a severely impaired pattern in both domains. Cluster 2 emerges from the patients who had a pronounced deficit in linguistic processing but less of an impairment in the non-linguistic domain. Five severely aphasic patients fell into this cluster; four are Broca's aphasics, and one is a Wernicke's aphasic. Cluster 3 contains the majority of the patients and contains patients who had milder impairments in either domain. Patients in Cluster 2 were marginally significantly more severely impaired (mean $A Q=40.8$, S.E.M. $=5.2$ ) and significantly less fluent (mean WAB fluency score $=2.6$, S.E.M. $=0.6$ ) compared with patients in Cluster 3 (mean $\mathrm{AQ}=68.4$, S.E.M. $=9.8 ;$ mean fluency score $=6.5$, S.E.M. $=1.3 ; P=0.06$ and $P=0.02$, respectively). Within Cluster 3 , accuracy scores in linguistic and non-linguistic trials were significantly correlated $[r=0.42 ; P=0.05]$.

Note that we already reported above that aphasia severity has a significant interaction with Domain-consistent with this, the cluster analysis reveals a subset of relatively severely affected individuals who show a disproportionate impairment in the linguistic compared with the and nonlinguistic domain. The remaining patients show correlated deficits.

An analogous cluster analysis on RT data did not reveal theoretically interesting clusters. Cluster 1 consists of Wernicke's aphasic patient RS (marked by ' $*$ ' in Fig. 4b, see below for outlier analysis) who was very slow to respond, especially on the linguistic trials. Cluster 2 (marked by ' + ' in Fig. 4b) contains eight patients who were relatively slow to respond in general. This group contains five Broca's aphasics, two Wernicke's aphasics and one Anomic patient. Cluster 3 contains the remaining patients.

We carried out outlier analyses in an attempt to identify any individual subjects who may exhibit dissociations between the linguistic and non-linguistic domains. We followed the procedure outlined by Bates et al. (2003a) in order to pick out the outliers and calculated density ellipses using a confidence interval of 95\% (the ellipses shown in Fig. 4). This procedure uses the Mahalanobis distance and takes into account the correlation structure of the data as well as the individual scales (Bates et al., 2003a).

For accuracy, two subjects remained outside the ellipse and were identified as outliers as shown in Fig. 4a. Patient JB performed at chance in both linguistic and non-linguistic domains and was the poorest-performing subject in the sam- ple. The second outlier was patient MB who was disproportionately affected in the linguistic domain - his accuracy in action comprehension in the non-linguistic domain was $94.4 \%$ while he managed to answer correctly in only $59.3 \%$ of the linguistic trials. For RT, we identified two outliers, as can be seen in Fig. 4b. These were patient RS and patient DC. Both patients were slower to respond to the linguistic trials with RS's discrepancy being much more pronounced. In summary, outlier analyses revealed a few potential dissociations in this sample of aphasic patients: JB was at chance in both domains and did not exhibit a dissociation. On the other hand, patient MB's performance in action comprehension through reading was severely compromised while he performed much better in action comprehension in the nonlinguistic domain. The outliers identified for RT data also showed more impairment in the linguistic domain, although these should be interpreted with caution since they are not mirrored in the accuracy data and also because longer response latencies for reading comprehension was characteristic of the behavior of severe aphasics in general. No outliers were identified who were markedly more impaired in the non-linguistic action condition, and thus we have no evidence here for a double dissociation.

In a series of papers, Varney proposed a theory of impairments following left-hemisphere strokes that result from two determinants: a supralinguistic impairment which also affects nonverbal abilities, and specific disturbances in processing semantic information from a sensory modality (Varney, 1978, 1980, 1982; Varney \& Benton, 1982). In particular, Varney (1978) reported that patients who were deficient in pantomime recognition were also impaired in reading comprehension. Conversely, all patients who were intact in reading were also intact in processing pantomime. As mentioned above, reading comprehension was relatively more impaired compared with pantomime interpretation across our population as well: 21 patients' $z$-scores differed by more than one in the direction of more impairment in the linguistic domain. There were five patients who showed the reverse pattern and three for whom the $z$-score differences were less than one. Note that this distributional information is reported for ease of comparison with previous results and is not considered to be evidence for dissociations (which have already been discussed above with more appropriate analysis techniques which take cross-domain correlations in the data into account).

\subsection{Lesion location analyses}

We performed a lesion analysis to investigate the neural correlates of linguistic and non-linguistic action processing using VLSM. Here, we constructed VLSM $d$-maps. Three axial slices for pantomime and reading comprehension are shown in Fig. 5. The color of each voxel reflects the magnitude of the difference between the scores of patients whose lesions included that voxel and those whose lesions did not include that voxel, which suggests the extent to 
(a) Lesion correlates of non-linguistic (pantomime interpretation)deficits
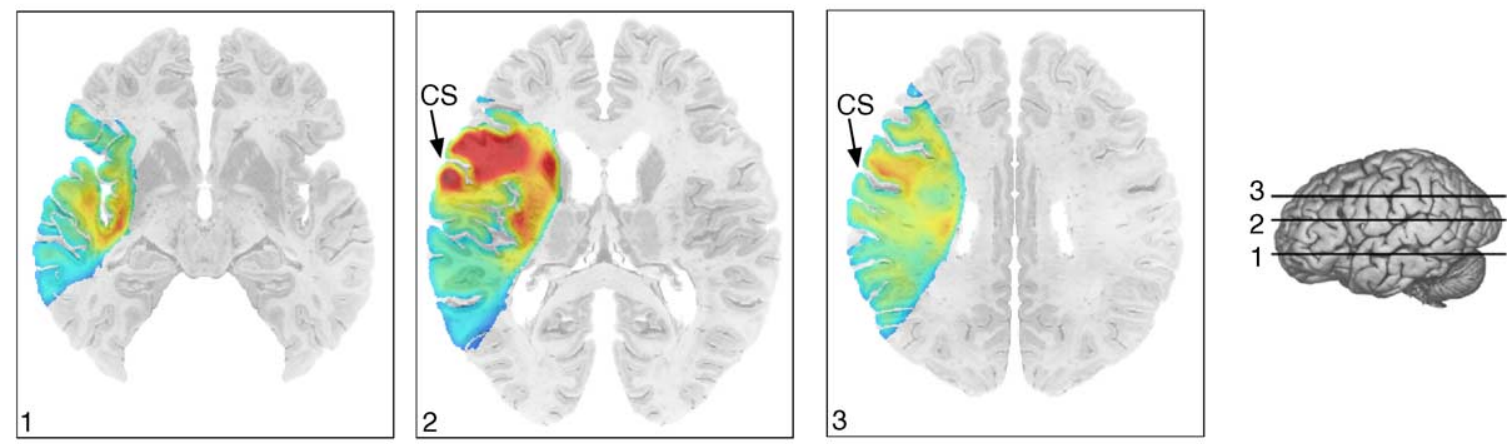

(b) Lesion correlates of linguistic (reading comprehension deficits)
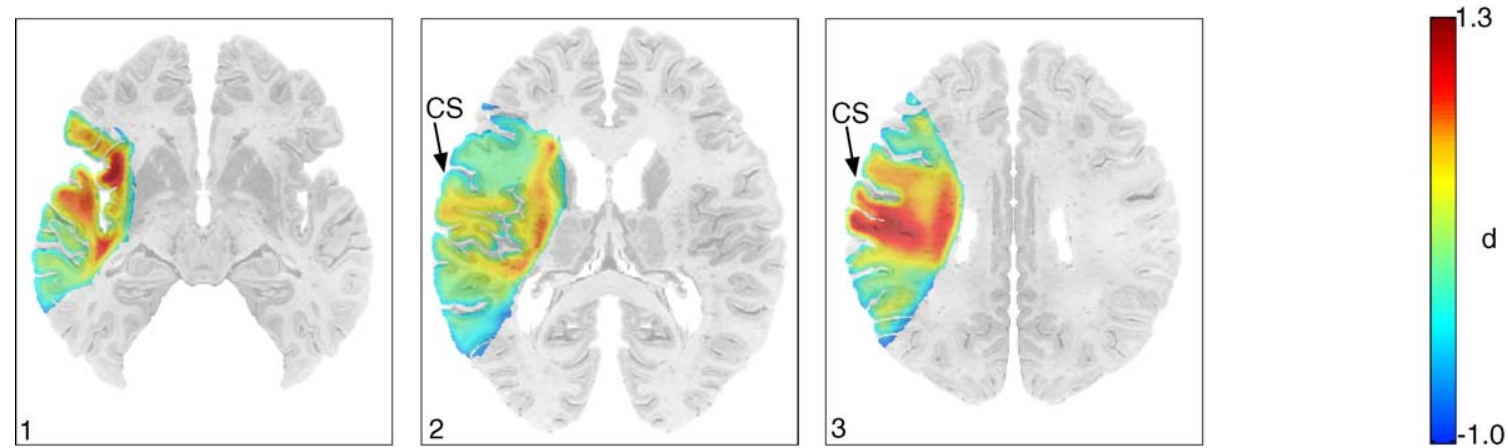

Fig. 5. Axial VLSM displays showing the relationship between tissue damage and behavioral deficits. The values displayed at each voxel are $d$-statistics comparing the patients lesioned at that voxel to the patients intact at that voxel. High $d$ values top the scale in red, indicating areas where damage led to significant deficits in task performance. Voxels denoted in blue reflect negative $d$ values, which arise when patients with lesions to those voxels performed better than those who had lesions elsewhere. Voxels that are not color-coded were damaged in less than 5 of the patients in our sample. The behavioral measures displayed are (a) non-linguistic action processing (pantomime interpretation), and (b) linguistic action processing (reading comprehension). The central sulcus (CS) is marked on slices 2 and 3, based on DeArmond et al.'s (1976) labeling in the atlas. The lateral view shows the approximate locations of the axial slices.

which damage to the voxel is associated with performance deficits.

The VLSM $d$-map for the accuracy measure in the nonlinguistic domain (Fig. 5a) revealed a large focal region including parts of the posterior inferior frontal gyrus (IFG) and ventral pre- and primary motor cortex (vPMC), extending medial to the frontal ventral-dorsal fibers of the superior longitudinal fasciculus (SLF), to be reliably associated with performance deficits when lesioned, as can be seen in slice 2. Also shown in red, just posterior and lateral to this area on the same slice, is part of the primary somatosensory cortex (PSC) in the postcentral gyrus. The head of the caudate nucleus is implicated, here visible again on slice 2. Finally, the most posterior focus shown in red on slice 2 includes white matter as well as part of the claustrum and possibly part of the insula, but the resolution does not permit us to distinguish between these structures. We will interpret these findings in more detail in the discussion.

The VLSM $d$-map for the accuracy measure in the linguistic domain (Fig. 5b) revealed several distinct areas where lesions were predictive of deficits in reading comprehension of actions. Deficits were associated with damage to the an- terior superior temporal gyrus (aSTG) extending back to the temporal isthmus, depicted on slice 1, the inferior anterior insula (aINS), also depicted on slice 1, and in the anterior inferior parietal lobe (aIPL) including parts of the postcentral and supramarginal gyri, as seen on slice 3 . Lesions affecting white matter were also associated with deficits: the internal capsule in slice 2 and the SLF in slice 3. These anatomical localizations were based largely on the sulcal and gyral labels in the DeArmond, Fusco, and Dewey (1976) atlas on which all patients' lesions were mapped.

Note that the lesion maps for linguistic versus nonlinguistic action comprehension deficits are quite distinct from one another, suggesting that different brain regions are important for these two tasks.

To analyze the lesion-symptom relationships in more detail, we chose six regions of interest (ROIs) based on these $d$ maps-points corresponding to maximal $d$-values in each of the "hot spots" in Fig. 5. The accuracy scores in the linguistic and non-linguistic domains of patients who have damage in these ROIs were compared to those whose lesions spared that ROI. This enabled us to quantitatively assess whether the areas we found in the $d$-maps are differentially impli- 
Table 3

Summary of region of interest (ROI) analyses

\begin{tabular}{|c|c|c|c|c|c|c|c|c|}
\hline \multirow[t]{2}{*}{ ROI } & \multirow[t]{2}{*}{ Brodmann areas } & \multicolumn{3}{|c|}{ Talairach coordinates } & \multicolumn{2}{|c|}{ Linguistic } & \multicolumn{2}{|c|}{ Non-linguistic } \\
\hline & & $x$ & $y$ & $z$ & $F$ & $P$ & $F$ & $P$ \\
\hline IFG/vPMC & $6,44,4$ & -48 & 10 & 16 & 0.04 & 0.42 & 5.09 & 0.018 \\
\hline PSC & $43,3,1,2$ & -60 & -10 & 16 & 0.13 & 0.36 & 7.83 & 0.006 \\
\hline CAU & - & -12 & 12 & 16 & 0.00 & 0.99 & 3.827 & 0.033 \\
\hline aSTG & 22,38 & -50 & 15 & 12 & 5.00 & 0.018 & 0.21 & 0.33 \\
\hline aINS & (13) & -37 & 10 & -6 & 7.54 & 0.006 & 0.00 & 0.50 \\
\hline aIPL & $40,3,1,2$ & -56 & -30 & 26 & 10.5 & 0.002 & 0.09 & 0.38 \\
\hline
\end{tabular}

Bold values indicate significant comparisons $(P<0.05)$.

cated in linguistic versus non-linguistic processing. Our six ROIs were the posterior part of the inferior frontal gyrus and pre- and primary motor cortex (IFG/vPMC), the portion of primary somatosensory cortex just posterior to IFG/vPMC (PSC), and the caudate head (CAU, all three ROIs based on Fig. 5a, slice 2), the anterior superior temporal gyrus (aSTG; based on Fig. 5b, slice 1), the inferior anterior insula (aINS; based on Fig. 5b, slice 1), the supramarginal gyrus and surrounding sensory cortex (aIPL; based on Fig. 5b, slice 3). Note that the aIPL and IFG/vPMC ROIs likely contain more than one anatomical region as it was not possible to obtain higher resolution inside these areas in this sample of patients given the distribution of their lesions. Table 3 depicts each ROI, associated Brodmann areas and approximate Talairach coordinates, here reported for comparison with other lesion or functional neuroimaging studies.

As could be expected based on the VLSM $d$-maps, lesions in the IFG/vPMC, PSC, CAU regions were associ- ated with significant deficits in pantomime interpretation. In these regions, there were no effects on reading comprehension (Fig. 6 and Table 3). We see the opposite pattern in the aSTG, aINS, aIPL: Lesions in these ROIs significantly affected reading comprehension of action information but did not have any effect in the non-linguistic domain (Fig. 6 and Table 3). Thus, the regions identified by our VLSM analyses and depicted in Fig. 5a and b are distinct areas and when damaged, have detrimental effects in performance in one domain but not the other.

Finally we examined the correlations between lesion volume and accuracy in the linguistic and non-linguistic action comprehension tasks. In our patient set, lesion volume varied greatly, from 6.4 to $162.6 \mathrm{cc}$, with a mean of $63.6 \mathrm{cc}$. However, lesion volume did not predict task performance in either of the two domains $[r=-0.03, P=0.90$ for accuracy in pantomime interpretation and $r=-0.26, P=0.25$ for reading comprehension].
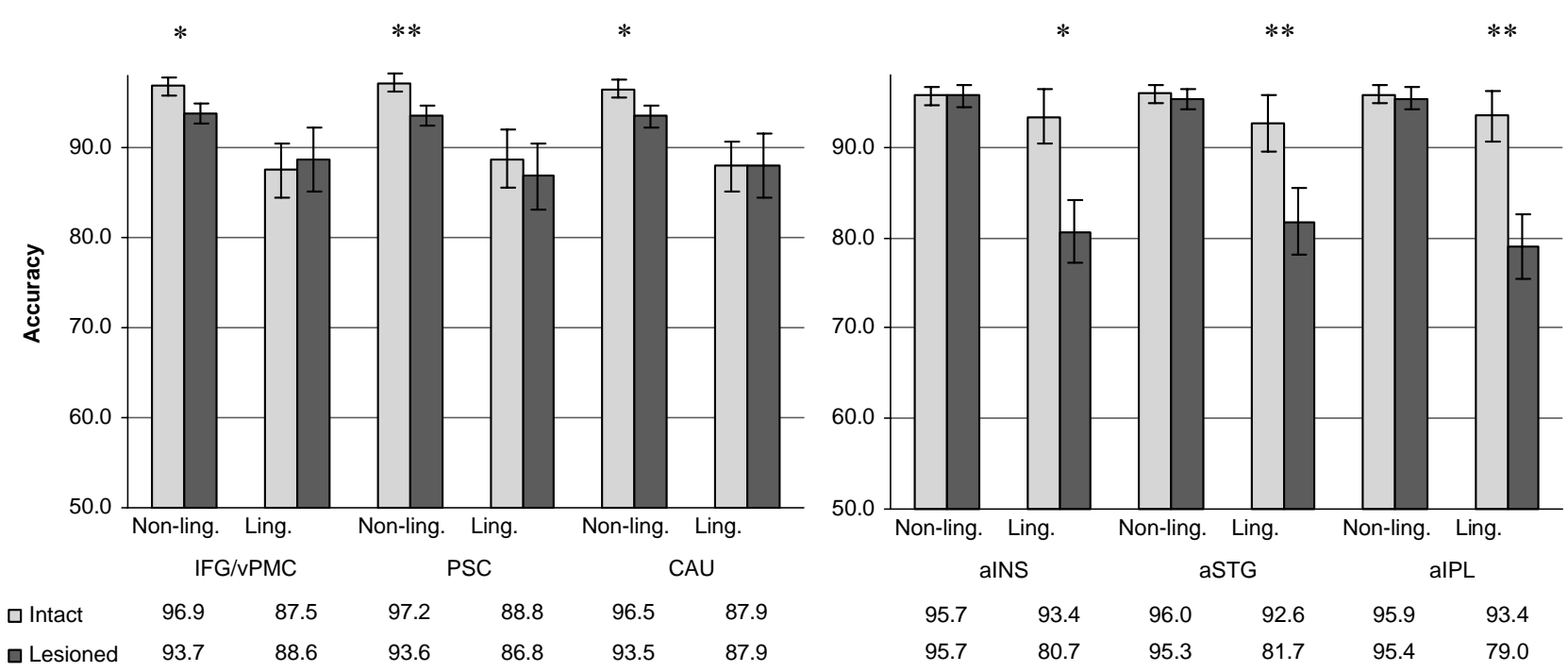

Fig. 6. Summary of statistics on the regions of interest: IFG/vPMC, PSC and CAU on the left graph and aSTG, aINS, and aIPL on the right. The IFG/vPMC and PSC foci were associated with significant deficits in the non-linguistic domain but not in the linguistic domain; conversely the aSTG, aINS and aIPL foci lesions caused significant impairments in linguistic, but not non-linguistic processing. ${ }^{*} P<0.05$; ${ }^{* *} P<0.01$ one-tailed-see Table 3 for more detail. Note that the absolute differences between scores of lesioned patients and intact patients are greater for the linguistic condition, but so are the associated error bars, reflecting higher variance in the sample for linguistic scores compared to non-linguistic scores. 


\section{Discussion}

\subsection{Action processing impairments in aphasia and their relation to language deficits}

Aphasic patients were significantly impaired in our actionto-picture matching task compared with control subjects. Performance was compromised in both linguistic and nonlinguistic domains. However, patients tended to show deficits that were more pronounced in the linguistic domain and the severity of aphasia was strongly related to the relative disparity between performance in linguistic and non-linguistic domains. There was no overall correlation between patients' deficits in the two domains, suggesting that the deficits observed in comprehension of pantomimed actions and comprehension of actions through reading are not tightly coupled processes.

Although global correlations in the dataset were not found, we have to refrain from concluding that action understanding in linguistic and non-linguistic domains are completely independent because we also found several pieces of evidence pointing to some shared substrates between linguistic and non-linguistic action understanding, which may help explain correlations observed in prior studies. Firstly, a large cluster of patients with relatively mild and relatively fluent aphasia did show correlated impairments in the two domains indicating perhaps there is some underlying relationship between these two tasks which does not hold for severely impaired subjects. Secondly, our outlier analyses taking correlation structure of the dataset into account did not lead to the identification of a significant number of individual patients exhibiting dissociations between the two domains and no double dissociations. Thirdly, the distracter manipulation showed no difference between the two domains, indicating common underlying processes, most likely of conceptual nature (see below).

What kind of conclusions can be drawn on the nature of non-linguistic deficits accompanying aphasia based on these results? We must reject a strong version of asymbolia, because pantomime comprehension impairments were not tightly correlated with linguistic deficits. On the other hand, we cannot hold that aphasia is a domain-specific disorder, because non-linguistic impairments are found in aphasia, and sometimes these are correlated with language deficits. Even in the present study, we see some evidence for some common substrates between linguistic and non-linguistic processing of action information. We must conclude then that the nature of the relationship between linguistic and nonlinguistic tasks or processes in question can be variable. Our view is that the more the non-linguistic task has in common with the linguistic task (e.g., in terms of perceptual similarity, conceptual networks involved, developmental stages the skills are acquired), the more likely they will be to have common brain areas subserving them, leading to correlated deficits in aphasic populations.

\subsection{Effects of semantically related and affordance-based distracters}

Three classes of distractors were employed in this study: semantic, affordance-based, and unrelated. Patients with aphasia were affected dramatically by semantic distracters, indicating that conceptual/semantic processes were especially compromised. Both patients and controls also made more errors when affordance-based distractors were present compared with unrelated distractors, although this did not reach significance for the patient group.

Semantic distracters are well known to affect aphasic patients' performance in non-linguistic domains, specifically in gesture and pantomime comprehension (Duffy \& Watkins, 1984; Seron et al., 1979; Varney, 1978; Varney \& Benton, 1982), consistent with our findings. Prior results for affordance-based distracters are less consistent: some studies have found that aphasic patients make more semantic and affordance-based errors compared with neutral errors in pantomime interpretation (Seron et al., 1979; Wang \& Goodglass, 1992); in another study more affordancebased than semantic errors were observed (Bell, 1994). We observed both kinds of errors, but semantic errors were considerably more frequent.

A few aspects of the distracter effects obtained in the present study were unexpected. First, even for the task of matching an object to a pictured action, semantic relatedness was a more potent distracter than affordance-based relatedness. We also collected data from college-age control subjects and verified the strong effect of semantic distracters (data not shown). We can conclude that associative or conceptual processes must be engaged in the comprehension of the action stimuli in both domains, at least in the context of this task. Thus both modality-specific and conceptual processes must be engaged in our task (see Glaser, 1992 for an argument that this is typical for conceptual tasks involving either words or pictures). The relatively small effect of affordance-based distracters remains more elusive and may need to be explored in further studies. Importantly, distracter-related effects did not differ across the verbal and nonverbal domains, suggesting that underlying processing deficits in aphasia have semantic/conceptual and affordancebased components that are not domain-specific.

\subsection{Lesion correlates of impairments in the non-linguistic domain}

We found that action processing deficits in the linguistic and non-linguistic domains have distinct lesion correlates. This section and the next discuss brain areas where lesions were predictive of non-linguistic and linguistic action comprehension deficits, respectively.

Deficits in non-linguistic action comprehension were associated with lesions in the inferior frontal and precentral gyri, in the primary somatosensory cortex in the postcentral gyrus, and in the head of the caudate. It is interesting that 
premotor and motor regions in the IFG/vPMC, known to be important for motor action production, were found also to be important for visual action comprehension. There was also involvement of the basal ganglia, specifically the caudate, which is another region involved in motor planning and control (see Caplan et al., 1990). The PSC area implicated is densely interconnected with pre- and primary motor cortex.

We believe these findings lend support to an embodied cognition view of action processing as they point to an underlying analysis-by-synthesis system. According to this view, an individual can understand others' actions by mapping the visual representation of the observed action onto his/her motor representation of the same action, thus using his/her own embodied experience of the world. In other words, "an action is understood when its observation causes the motor system of the observer to 'resonate"' (Rizzolatti, Fogassi, \& Gallese, 2001; p. 661; see also Jeannerod, 1995, 2001).

Recently, the discovery of the "mirror neuron system" has added a new dimension to research concerning the neural representation of action. Mirror neurons are a particular class of visuo-motor neurons that were first found in area F5 in the ventral premotor cortex of the macaque (Gallese, Fadiga, Fogassi, \& Rizzolatti, 1996; Rizzolatti, Fadiga, Gallese, \& Fogassi, 1996a). The main functional characteristic of these neurons is that they fire not only when an animal executes a particular action, but also when the animal observes another individual performing the same or a similar action. The existence of a similar mirror system in humans has been demonstrated by a variety of neurophysiological and neuroimaging studies, revealing neural activity in premotor and inferior frontal cortical areas (as part of a larger network involving superior temporal and parietal regions) during action observation and imitation (e.g., Buccino et al., 2004; Fadiga, Fogassi, Pavesi, \& Rizzolatti, 1995; Grafton, Arbib, Fadiga, \& Rizzolatti, 1996; Grèzes, Armony, Rowe, \& Passingham, 2003; Iacoboni et al., 1999; Rizzolatti et al., 1996b), and even during viewing of manipulable objects (e.g., Chao \& Martin, 2000). Another interesting aspect of the human mirror neuron system is that the responses in frontal cortex during action observation have been fairly consistently left-lateralized in different studies (e.g., Grafton et al., 1996; Grèzes et al., 2003; Iacoboni et al., 1999; Rizzolatti et al., 1996b), consistent with findings from the neuropsychological literature on the dominance of this hemisphere for action processing.

These findings are in agreement with our analysis of the lesion correlates of non-linguistic action processing. Indeed, embodied action representation theories may help explain the roles of not only the IFG/vPMC regions, but also the other areas which were predictive of deficits: there is evidence that this kind of embodied perception may also involve somatosensory regions of the brain (see Avikainen, Forss, \& Hari, 2002; Keysers et al., 2004). Another fMRI study has also found that the caudate is active during imagery of hand motions (Gerardin et al., 2000). Thus, we argue that lesions to the IFG/vPMC, PSC and CAU were associated with poor performance in our non-linguistic action comprehension task because processing in this embodied network was disrupted in our patients by damage to parts of this analysis-by-synthesis system.

One potentially relevant role of left frontal areas in action comprehension is the view that these regions may be important for action naming and/or verb processing (e.g. Perani et al., 1999). However, as noted above, some studies have not found differences between verb and noun processing (e.g. Tyler et al., 2001), and Hillis et al.'s (2002) study with neuropsychological patients found evidence for left frontal involvement only for the naming of actions, but not for their comprehension.

There have been relatively few prior neuropsychological studies of action comprehension which have attempted to identify relevant brain areas. This is partly because deficits in movement production (apraxia) have been more studied than deficits in action comprehension. An important focus of the literature on apraxia is the role of the parietal lobe. In a recent review, Koski, Iacoboni, and Mazziotta (2002) concluded that "the left parietal cortex subserves a particularly important component of the praxis system, especially concerned with the knowledge or representation of overlearned actions" (p. 75). As mentioned in the introduction, apraxic patients with posterior lesions have been reported to have more trouble not only in action production, but also in comprehending the meaning of pantomimes (Heilman et al., 1982; Rothi et al., 1985). There is also evidence that parietal lesions may be detrimental to the perception of biological motion (Battelli, Cavanagh, \& Thornton, 2003; Saygin, Wilson, Hagler, Bates, \& Sereno, 2003b).

In contrast to these findings of the importance of parietal areas for action understanding, Halsband et al. (2001) examined parietal and premotor-lesioned patients and found that while patients with left parietal damage were most impaired in imitation of pantomimes, they did not show differential comprehension deficits. Likewise we did not observe parietal lesions (except for the small locus in the PSC) to be associated with pantomime interpretation deficits. A possible reason for this could be the stationary nature of our stimuli, as parietal areas are known to be involved in visuo-motor transformations. Although stationary images with implied motion or action can activate motion-sensitive areas in functional neuroimaging studies (e.g., Kourtzi \& Kanwisher, 2000), these activations are usually in the occipital and temporal regions.

A parallel finding to ours from the neuropsychological literature has very recently been reported by Tranel et al. (2003): Their lesion-symptom mapping procedure identified very similar left inferior frontal areas (along with parietal and temporal regions) to be associated with deficits in conceptual knowledge of actions in a group of patients. It is not unexpected for our study to have some different lesion findings with Tranel et al.'s as the tasks administered were different in the two studies. However, the common frontal lesion finding probably reflects the neural regions subserv- 
ing shared processes involved in action understanding and retrieving conceptual knowledge for actions. We believe both Tranel's results and ours are beginning to show that these frontal regions may be important lesion correlates for action processing in the nonverbal domain.

\subsection{Lesion correlates of impairments in the linguistic domain}

We found three regions that are associated with impairments in the linguistic domain in our task (but not in the non-linguistic domain): aSTG, aINS, and aIPL.

The anterior temporal lobe has been implicated as an area that is important for sentence processing in previous neuropsychological work (Dronkers, Wilkins, Van Valin, Redfern, \& Jaeger, 2004). Neuroimaging studies have also pointed to the role of this region in sentence processing in both auditory (see Staab, 2002 for a review) and visual (Stowe et al., 1999; Vandenberghe, Nobre, \& Price, 2002) modalities. These results are consistent with our findings because the linguistic stimuli along with the completion task would be expected to rely upon sentence-level processing.

The superior anterior insula in the left hemisphere has also been identified by Dronkers (1996) as a crucial area for language processing: Lesions in this part of the brain are associated with impairments in speech production. This finding has received further support from subsequent neuropsychological (Bates et al., 2003b) and neuroimaging studies (e.g., Blank, Scott, Murphy, Warburton, \& Wise, 2002). The region we found in the present study is slightly inferior to the part of the insula reported in Dronkers (1996). In a recent fMRI study, the insula was among the regions that showed increased activity for "tongue-twister" sentences, even though the task was reading comprehension and did not involve articulation (Keller, Carpenter, \& Just, 2003). We believe the involvement of this region in deficits in the linguistic domain in our experiment is most likely due to a recoding of read material into phonological and/or articulatory representations (e.g., Coltheart et al., 1993; Plaut et al., 1996).

The aIPL area identified for linguistic action comprehension deficits may reflect the involvement of either linguistic or sensorimotor systems. This region overlaps partially with the supramarginal gyrus which is known to be important for a number of linguistic functions including phonological (Fujimaki et al., 1999) and semantic processing (Bullmore et al., 1996; Metter et al., 1990). A large group study of aphasic patients found that the supramarginal gyrus (along with the posterior middle and superior temporal gyri) were most often damaged in patients with reading comprehension deficits (Hojo, Watanabe, Tasaki, Sato, \& Metoki, 1985). This region could also be important for the conversion of orthography to phonology (Booth et al., 2002; Moore \& Price, 1999) and may be part of the "articulatory loop" for verbal working memory (Paulesu, Frith, \& Frackowiak, 1993).

On the other hand, this region of parietal cortex is also known to contain mirror neurons in the macaque (see
Rizzolatti et al., 2001) and recent human studies have provided evidence that it is a component of the human mirror neuron system as well (Buccino et al., 2004), showing activation in areas very close to the ones found in our VLSM maps. Thus the involvement of the aIPL focus in our lesion map for reading comprehension may also reflect embodied action comprehension processes. Indeed, based on the relatively anterior location of this lesion focus, it may be more likely that this lesion site reflects the involvement of the mirror neuron system, rather than the linguistic systems discussed above, which tend to be associated with more posterior portions of the inferior parietal lobule. In this latter interpretation, however, it is interesting that action comprehension in the linguistic modality may rely selectively upon the parietal component of the mirror neuron circuitry, while in the non-linguistic modality we see the selective involvement of the frontal component (see Fig. 6). Further studies, perhaps with neuroimaging, may shed more light on why our pantomime interpretation task requires access to the frontal subpart of the mirror neuron system while the linguistic action comprehension task may require access to the parietal subpart.

The fact that we found multiple lesion foci to be associated with deficits in reading comprehension of actions is perhaps not unexpected given that in this task, there could be different components to the impairment in the linguistic domain-i.e., there may be potentially independent factors at play such as an inability to understand written sentences, or deficits in matching the actions described in text to corresponding objects, or a difficulty with processing the action information itself. But the effects of these different factors would be compounded in the behavioral scores and associated lesion sites. Thus, it is possible that the different ROIs we found are associated with different aspects of the task. Based on prior work however, we propose that the aSTG involvement reflects sentence-level linguistic processing aspects of the task, while the aINS (and perhaps aIPL) is involved in translating between different code systems during reading comprehension (orthographic, phonological, articulatory) and aIPL may additionally be involved in action understanding due to being part of the mirror neuron system.

\subsection{Theoretical discussion: neuropsychological evidence for embodied representations in action perception}

We propose that the lesion sites we identified in the present study support a view which is sometimes called embodied cognition, and here we discuss the lesion as well as the behavioral results from this theoretical perspective. The embodied cognition view emphasizes that the brain functions in a body, which in turn, develops and functions in an environment-both physical and social. Proponents of this view hold that this needs to be taken into account in order to understand the functional organization of the brain for different sensory, motor and cognitive domains and tasks. While similar ideas have been put forth by several pioneers 
in psychology (see Gibson, 1996, 1977; Werner \& Kaplan, 1967), most work in embodied cognition is relatively recent. Researchers working in this paradigm argue that seemingly abstract concepts in language and higher cognitive domains can be grounded onto a body-based framework (see, Barsalou, 1999; Lakoff \& Johnson, 1980) and a number of studies have reported behavioral evidence in support of this view (e.g., Gernsbacher, Varner, \& Faust, 1990; Glenberg $\&$ Kaschak, 2002).

The discovery of mirror neurons in the macaque, and findings suggesting a homologous system in humans, have been exciting developments for embodied cognition. This research has shown that areas of the brain which subserve motor action production are also involved in action perception and comprehension. Thus one's own body and action representations are used as templates and simulations in order to understand those of others. The present experiment now adds neuropsychological evidence to this body of literature, by showing that lesions in these premotor and motor areas can lead to deficits in the comprehension of information representing actions.

The lesion sites we identified which are not part of the mirror neuron network can also be understood within the framework of embodied cognition. Here, we argue that lesion sites we observed are related to the task components, rather than the semantics of the actions in the sentences (with the possible exception of the anterior parietal focus). The lesion sites which led to deficits in reading comprehension of actions are areas which are involved in sentence comprehension, phonological processing, and interestingly, speech planning and articulation. Note crucially that the embodiment view always takes development into account. By the time people learn how to read, spoken language has already been acquired, and there is already in place a rich multisensory, semantic representation of the world. Reading skills would thus be overlaid upon already existing neural circuitry for carrying out related linguistic and non-linguistic operations, rather than having its own domain-specific neural regions. Our results are in agreement with this kind of model.

Finally, note that an embodied cognition view is not at odds with the lack of correlation between domains observed in the behavioral results of the present study. A strong asymbolia view would expect such an outcome, but embodiment does not imply complete overlap of related processes. In this particular case, even though task and stimulus level factors were controlled for across the two modalities, there were other varying factors between the two domains. According to the embodied cognition view, the non-linguistic action comprehension system would be overlaid very early in development on the body's own motor, sensory and proprioceptive representational systems, whereas reading, being a later-acquired skill, would be overlaid on a more distributed linguistic and conceptual network. If the systems are acquired and related skills are honed at such different stages in development, the resulting brain networks subserving processing in the two domains will also be rather different, and patients with brain injury will not show tightly correlated deficits. In contrast, in a very similar study we conducted in the auditory modality, where the linguistic and nonlinguistic stimuli are both perceptually similar and are acquired at similar stages in development (Cummings, Saygin, Dick, $\&$ Bates, 2004), we did find tightly correlated deficits in aphasic patients' performance, along with shared lesion sites (Saygin et al., 2003a).

To summarize, patients with aphasia had globally uncorrelated deficits in the comprehension of action information through pantomime interpretation and reading comprehension. On the other hand, we also found evidence for some shared underlying processes. Patients had impairments in both pantomime interpretation and comprehension of actions through reading but their deficits were more pronounced in the linguistic domain, especially for the more severe aphasics. Pantomime interpretation deficits were associated with lesions in anterior brain areas known to be involved in motor planning and execution, demonstrating that lesions in the frontal component of the human mirror neuron system are associated with deficits in action understanding in left hemisphere injured patients. Reading comprehension deficits followed from damage to brain areas known to be involved in linguistic processes including sentence processing, speech articulation and phonological processing, and potentially also the parietal component of the human mirror neuron system.

\section{Acknowledgements}

We are grateful to H. Saygin for help with preparing the picture stimuli, R. Buffington, C. Ludy, S. Moineau, and E. Schleicher for assistance with testing or programming, R.T. Knight for lesion reconstructions, F. Dick, and M. Iacoboni for helpful discussions and all of our subjects for participating in our experiments. We also thank L. Barsalou, G. Goldenberg, the anonymous reviewers, and M. Moscovitch for their suggestions on previous versions of this manuscript, which greatly improved our work. This research was supported by NIDCD RO1 DC00216 to E. Bates and colleagues and Department of Veterans Affairs Medical Research and NINDS project grants to N. Dronkers and colleagues (PO1 NS17778 and PO1 NS40813).

\section{References}

Avikainen, S., Forss, N., \& Hari, R. (2002). Modulated activation of the human SI and SII cortices during observation of hand actions. NeuroImage, 15, 640-646.

Barsalou, L. W. (1999). Perceptual symbol systems. The Behavioral and Brain Sciences, 22, 577-609.

Bates, E., Appelbaum, M., Salcedo, J., Saygin, A. P., \& Pizzamiglio, L. (2003a). Quantifying dissociations in neuropsychological research. Journal of Experimental and Clinical Neuropsychology, 25, 1128-1153. 
Bates, E., Wilson, S. M., Saygin, A. P., Dick, F., Sereno, M. I., Knight, R., \& Dronkers, N. F. (2003b). Voxel-based lesion-symptom mapping. Nature Neuroscience, 6, 448-450.

Battelli, L., Cavanagh, P., \& Thornton, I. M. (2003). Perception of biological motion in parietal patients. Neuropsychologia, 41, 18081816.

Bell, B. D. (1994). Pantomime recognition impairment in aphasia: an analysis of error types. Brain and Language, 47, 269-278.

Blank, S. C., Scott, S. K., Murphy, K., Warburton, E., \& Wise, R. J. S. (2002). Speech production: Wernicke, Broca and beyond. Brain, 125, 1829-1838.

Booth, J. R., Burman, D. D., Meyer, J. R., Gitelman, D. R., Parrish, T. B., \& Mesulam, M. M. (2002). Functional anatomy of intra- and cross-modal lexical tasks. NeuroImage, 16, 7-22.

Buccino, G., Vogt, S., Ritzl, A., Fink, G. R., Zilles, K., \& Freund, H. J. et al., (2004). Neural circuits underlying imitation learning of hand actions: An event-related FMRI study. Neuron, 42, 323-334.

Bullmore, E. T., Rabe-Hesketh, S., Morris, R. G., Williams, S. C., Gregory, L., \& Gray, J. A. (1996). Functional magnetic resonance image analysis of a large-scale neurocognitive network. Neurolmage, 4, 16-33.

Caplan, L. R., Schmahmann, J. D., Kase, C. S., Feldmann, E., Baquis, G., \& Greenberg, J. P. (1990). Caudate infarcts. Archives of Neurology, 47, 133-143.

Chao, L. L., \& Martin, A. (2000). Representation of manipulable manmade objects in the dorsal stream. NeuroImage, 12, 478-484.

Cohen, J. D., MacWhinney, B., Flatt, M., \& Provost, J. (1993). PsyScope: An interactive graphic system for designing and controlling experiments in the psychology laboratory using Macintosh computers. Behavior Research Methods, Instruments, and Computers, 25, 257-271.

Coltheart, M., Brent, C., Atkins, P., \& Haller, M. (1993). Models of reading aloud: Dual-route and parallel-distributed-processing approaches. Psychological Review, 100, 589-608.

Cummings, A., Saygin, A. P., Dick, F., \& Bates, E. (2004). Is there a dissociation between verbal and environmental sound processing in young children? [Abstract]. In Proceedings of the 14th biennial international conference on infant studies, May 5-8, 2004, Chicago, IL.

De Renzi, E., Faglioni, P., Scarpa, M., \& Crisi, G. (1986). Limb apraxia in patients with damage confined to the left basal ganglia and thalamus. Journal of Neurology, Neurosurgery and Psychiatry, 49, 1030-1038.

De Renzi, E., Faglioni, P., Scotti, G., \& Spinnler, H. (1972). Impairment in associating colour to form, concomitant with aphasia. Brain, 95, 293-304.

De Renzi, E., Pieczuro, A., \& Vignolo, L. A. (1968). Ideational apraxia: A quantitative study. Neuropsychologia, 6, 41-52.

DeArmond, S. J., Fusco, M. M., \& Dewey, M. M. (1976). Structure of the human brain: A photographic atlas. New York: Oxford University Press.

Dronkers, N. F. (1996). A new brain region for coordinating speech articulation. Nature, 384, 159-161.

Dronkers, N. F., Wilkins, D. P., Van Valin Jr., R. D., Redfern, B. B., \& Jaeger, J. J. (2004). Lesion analysis of the brain areas involved in language comprehension. Cognition, 92, 145-177.

Duffy, R. J., \& Duffy, J. R. (1981). Three studies of deficits in pantomimic expression and pantomime recognition in aphasia. Journal of Speech and Hearing Research, 46, 70-84.

Duffy, J. R., \& Watkins, L. B. (1984). The effect of response choice relatedness on pantomime and verbal recognition ability in aphasic patients. Brain and Language, 21, 291-306.

Fadiga, L., Fogassi, L., Pavesi, G., \& Rizzolatti, G. (1995). Motor facilitation during action observation: a magnetic stimulation study. Journal of Neurophysiology, 73, 2608-2611.

Ferro, J. M., Martins, I. P., Mariano, G., \& Castro Caldas, A. C. (1983). CT scan correlates of gesture recognition. Journal of Neurology, Neurosurgery and Psychiatry, 46, 943-952.

Finkelnburg, D. C. (1870). [Niederrheinische Gesellschaft in Bonn, Sitzung vom 21. März 1870.] Berliner Klinische Wochenschrift, 7, 449-450, 460-462.
Fujimaki, N., Miyauchi, S., Putz, B., Sasaki, Y., Takino, R., \& Sakai, K. (1999). Functional magnetic resonance imaging of neural activity related to orthographic, phonological, and lexico-semantic judgments of visually presented characters and words. Human Brain Mapping, 8, $44-59$.

Gainotti, G., \& Lemmo, M. S. (1976). Comprehension of symbolic gestures in aphasia. Brain and Language, 3, 451-460.

Gallese, V., Fadiga, L., Fogassi, L., \& Rizzolatti, G. (1996). Action recognition in the premotor cortex. Brain, 119, 593-609.

Gerardin, E., Sirigu, A., Lehericy, S., Poline, J. B., Gaymard, B., \& Marsault, C. (2000). Partially overlapping neural networks for real and imagined hand movements. Cerebral Cortex, 10, 1093-1104.

Gernsbacher, M. A., Varner, K. R., \& Faust, M. E. (1990). Investigating differences in general comprehension skill. Journal of Experimental Psychology: Learning, Memory, \& Cognition, 16, 430-445.

Gibson, J. J. (1996). The senses considered as perceptual systems. Boston: Houghton Mifflin.

Gibson, J. J. (1977). The theory of affordances. In: R. E. Shaw \& J. Bransford (Eds.), Perceiving, acting, and knowing. Hillsdale, NJ: Lawrence Erlbaum.

Glaser, W. R. (1992). Picture naming. Cognition, 42, 61-106.

Glenberg, A. M., \& Kaschak, M. P. (2002). Grounding language in action. Psychonomic Bulletin \& Review, 9, 558-569.

Goldenberg, G. (1999). Matching and imitation of hand and finger postures in patients with damage in the left or right hemispheres. Neuropsychologia, 37, 559-566.

Goldstein, K. (1948). Language and language disturbances: Aphasic symptom complexes and their significance for medicine and theory of language. New York: Grune \& Stratton.

Goodglass, H., \& Kaplan, E. (1963). Disturbance of gesture and pantomime in aphasia. Brain, 86, 703-720.

Grafton, S. T., Arbib, M. A., Fadiga, L., \& Rizzolatti, G. (1996). Localization of grasp representations in humans by PET. 2. Observation compared with imagination. Experimental Brain Research, 112, 103111.

Grèzes, J., Armony, J. L., Rowe, J., \& Passingham, R. E. (2003). Activations related to "mirror" and "canonical" neurones in the human brain: an fMRI study. NeuroImage, 18, 928-937.

Halsband, U., Schmitt, J., Weyers, M., Binkofski, F., Grutzner, G., \& Freund, H. J. (2001). Recognition and imitation of pantomimed motor acts after unilateral parietal and premotor lesions: A perspective on apraxia. Neuropsychologia, 39, 200-216.

Head, H. (1926). Aphasia and kindred disorders of speech. Cambridge: Cambridge University Press.

Heilman, K. M., Rothi, L. J., \& Valenstein, E. (1982). Two forms of ideomotor apraxia. Neurology, 32, 342-346.

Hillis, A. E., Tuffiash, E., Wityk, R. J., \& Barker, P. B. (2002). Regions of neural dysfunction associated with impaired naming of actions and objects in acute stroke. Cognitive Neuropsychology, 19, 523-534.

Hojo, K., Watanabe, S., Tasaki, H., Sato, T., \& Metoki, H. (1985). Localization of lesions in aphasia: clinical-CT scan correlations (part II). No To Shinkei, 37, 81-88.

Iacoboni, M., Woods, R. P., Brass, M., Bekkering, H., Mazziotta, J. C., \& Rizzolatti, G. (1999). Cortical mechanisms of human imitation. Science, 286, 2526-2528.

Jeannerod, M. (1995). Mental imagery in the motor context. Neuropsychologia, 33, 1419-1432.

Jeannerod, M. (2001). Neural simulation of action: A unifying mechanism of motor cognition. Neurolmage, 14, S103-S109.

Keller, T. A., Carpenter, P. A., \& Just, M. A. (2003). Brain imaging of tongue-twister sentence comprehension: Twisting the tongue and the brain. Brain and Language, 84, 189-203.

Kertesz, A. (1979). Aphasia and associated disorders: Taxonomy, localization, and recovery. New York: Grune \& Stratton.

Kertesz, A., Ferro, J. M., \& Shewan, C. M. (1984). Apraxia and aphasia: The functional-anatomical basis for their dissociation. Neurology, 34, $40-47$. 
Keysers, C., Wicker, B., Gazzola, V., Anton, J. L., Fogassi, L., \& Gallese, V. (2004). A touching sight: SII/PV activation during the observation and experience of touch. Neuron, 42, 335-436.

Kimura, D. (1977). Acquisition of a motor skill after left-hemisphere damage. Brain, 100, 527-542.

Koski, L., Iacoboni, M., \& Mazziotta, J. C. (2002). Deconstructing apraxia: Understanding disorders of intentional movement after stroke. Current Opinion in Neurology, 15, 71-77.

Kourtzi, Z., \& Kanwisher, N. (2000). Activation in human MT/MST by static images with implied motion. Journal of Cognitive Neuroscience, $12,48-55$.

Lakoff, G., \& Johnson, M. (1980). Metaphors we live by. Chicago: University of Chicago Press.

Landauer, T. K., Foltz, P. W., \& Laham, D. (1998). An introduction to latent semantic analysis. Discourse Processes, 25, 259-284.

Metter, E. J., Hanson, W. R., Jackson, C. A., Kempler, D., van Lancker, D., \& Mazziotta, J. C. (1990). Temporoparietal cortex in aphasia: Evidence from positron emission tomography. Archives of Neurology, 47, 1235-1238.

Moore, C. J., \& Price, C. J. (1999). Three distinct ventral occipitotemporal regions for reading and object naming. NeuroImage, 10, 181-192.

Paulesu, E., Frith, C. D., \& Frackowiak, R. S. (1993). The neural correlates of the verbal component of working memory. Nature, 362, 342-345.

Perani, D., Cappa, S. F., Schnur, T., Tettamanti, M., Collina, S., \& Rosa, M. M. (1999). The neural correlates of verb and noun processing. A PET study. Brain, 122, 2337-2344.

Pickett, L. W. (1974). An assessment of gestural and pantomimic deficit in aphasic patients. Acta Symbolica, 5, 69-86.

Plaut, D. C., McClelland, J. L., Seidenberg, M. S., \& Patterson, K. (1996). Understanding normal and impaired word reading: Computational principles in quasi-regular domains. Psychological Review, 103, 56115.

Rizzolatti, G., Fadiga, L., Gallese, V., \& Fogassi, L. (1996a). Premotor cortex and the recognition of motor actions. Cognitive Brain Research, $3,131-141$

Rizzolatti, G., Fadiga, L., Matelli, M., Bettinardi, V., Paulesu, E., \& Perani, D. (1996b). Localization of grasp representations in humans by PET. 1. Observation versus execution. Experimental Brain Research, $111,246-252$.

Rizzolatti, G., Fogassi, L., \& Gallese, V. (2001). Neurophysiological mechanisms underlying the understanding and imitation of action. Nature Reviews Neuroscience, 2, 661-670.

Rothi, L. J., Heilman, K. M., \& Watson, R. T. (1985). Pantomime comprehension and ideomotor apraxia. Journal of Neurology, Neurosurgery and Psychiatry, 48, 207-210.

Saygin, A. P., Dick, F., Wilson, S., Dronkers, N., \& Bates, E. (2003a). Neural resources for processing language and environmental sounds: Evidence from aphasia. Brain, 126, 928-945.

Saygin, A. P., Wilson, S. M., Hagler Jr., D. J., Bates, E., \& Sereno, M. I. (2003b). Brain areas involved in the processing of biological motion:
Lesion-symptom mapping and fMRI [Abstract]. In Proceedings of the 9th international conference on functional mapping of the human brain, June 19-22, 2003, New York, NY. Available on CD-ROM in NeuroImage, 19(2)

Schnider, A., Hanlon, R. E., Alexander, D. N., \& Benson, D. F. (1997). Ideomotor apraxia: Behavioral dimensions and neuroanatomical basis. Brain and Language, 58, 125-136.

Schuell, H. (1965). Differential diagnosis of aphasia with the Minnesota test. Minneapolis: University of Minnesota Press.

Seron, X., van der Kaa, M. A., Remitz, A., \& van der Linden, M. (1979). Pantomime interpretation and aphasia. Neuropsychologia, 17, 661-668.

Sokal, R. R., \& Sneath, P. H. A. (1973). Numerical taxonomy: The principles and practice of numerical classification. London: Freeman.

Spinnler, H., \& Vignolo, L. A. (1966). Impaired recognition of meaningful sounds in aphasia. Cortex, 2, 337-348.

Staab, J. (2002). On the role of the anterior superior temporal lobe in language processing: Hints from functional neuroimaging studies. Center for Research in Language Newsletter, 14(3), 3-14.

Stowe, L. A., Paans, A. M., Wijers, A. A., Zwarts, F., Mulder, G., \& Vaalburg, W. (1999). Sentence comprehension and word repetition: a positron emission tomography investigation. Psychophysiology, 36, 786-801.

Szekely, A., D’Amico, S., Devescovi, A., Federmeier, K., Herron, D., \& Iyer, G. (2004). Timed picture naming: Extended norms and validation against previous studies. Behavior Research Methods Instruments Computers, 35, 621-633.

Tranel, D., Kemmerer, D., Damasio, H., Adolphs, R., \& Damasio, A. R. (2003). Neural correlates of conceptual knowledge for actions. Cognitive Neuropsychology, 20, 409-432.

Tyler, L. K., Russell, R., Fadili, J., \& Moss, H. E. (2001). The neural representation of nouns and verbs: PET studies. Brain, 124, 1619-1634.

Vandenberghe, R., Nobre, A. C., \& Price, C. J. (2002). The response of left temporal cortex to sentences. Journal of Cognitive Neuroscience, $14,550-560$.

Varney, N. R. (1978). Linguistic correlates of pantomime recognition in aphasic patients. Journal of Neurology, Neurosurgery and Psychiatry, $41,564-568$

Varney, N. R. (1980). Sound recognition in relation to aural language comprehension in aphasic patients. Journal of Neurology, Neurosurgery and Psychiatry, 43, 71-75.

Varney, N. R. (1982). Pantomime recognition defect in aphasia: Implications for the concept of asymbolia. Brain and Language, 15, 32-39.

Varney, N. R., \& Benton, A. L. (1982). Qualitative aspects of pantomime recognition defect in aphasia. Brain and Cognition, 1, 132-139.

Wang, L., \& Goodglass, H. (1992). Pantomime, praxis, and aphasia. Brain and Language, 42, 402-418.

Werner, H., \& Kaplan, B. (1967). Symbol formation: An organismicdevelopmental approach to language and expression of thought (3rd ed.). New York: Wiley. 